EPJ Web of Conferences 64, 06003 (2014)

DOI: $10.1051 /$ epjconf/ 20146406003

(C) Owned by the authors, published by EDP Sciences, 2014

\title{
Luminosity dependent accretion state change in GRO J1008-57
}

Matthias Kühnel ${ }^{1, a}$, Sebastian Müller ${ }^{1}$, Ingo Kreykenbohm ${ }^{1}$, Felix Fürst ${ }^{2}$, Katja Pottschmidt ${ }^{3,4}$, Richard E. Rothschild ${ }^{5}$, Isabel Caballero ${ }^{6}$, Victoria Grinberg ${ }^{1}$, Gabriele Schönherr ${ }^{7}$, Chris Shrader $^{4,8}$, Dmitry Klochkov ${ }^{9}$, Rüdiger Staubert ${ }^{9}$, Carlo Ferrigno ${ }^{10}$, José-Miguel Torrejón ${ }^{11}$, Silvia Martínez-Núñez ${ }^{11}$, and Jörn Wilms ${ }^{1}$

${ }^{1}$ Dr. Karl Remeis-Observatory \& ECAP, Universität Erlangen-Nürnberg, Sternwartstr. 7, 96049 Bamberg, Germany

${ }^{2}$ Space Radiation Lab, California Institute of Technology, MC 290-17 Cahill, 1200 E. California Blvd., Pasadena, CA 91125, USA

${ }^{3}$ CRESST, Center for Space Science and Technology, UMBC, Baltimore, MD 21250, USA

${ }^{4}$ NASA Goddard Space Flight Center, Greenbelt, MD 20771, USA

${ }^{5}$ Center for Astronomy and Space Sciences, University of California, San Diego, La Jolla, CA 92093, USA

${ }^{6}$ CEA Saclay, DSM/IRFU/SAp-UMR AIM (7158) CNRS/CEA/Université Paris 7, Diderot, 91191 Gif sur Yvette, France

${ }^{7}$ Leibniz-Institut für Astrophysik Potsdam, An der Sternwarte 16, 14482 Potsdam, Germany

${ }^{8}$ Universities Space Research Association, Columbia, MD 21044, USA

9 Institut für Astronomie und Astrophysik, Universität Tübingen, Sand 1, 72076 Tübingen, Germany

${ }^{10}$ ISDC Data Center for Astrophysics, Chemin d'Écogia 16, 1290 Versoix, Switzerland

${ }^{11}$ Instituto de Física Aplicada a las Ciencias y las Tecnologías, Universidad de Alicante, 03080 Alicante, Spain

\begin{abstract}
In a former publication, we have analyzed the transient neutron star X-ray binary GRO J1008-57 using all available RXTE-, Swift-, and Suzaku-data. As we have found, the source's spectral components, i.e., a power-law with high exponential cutoff and a black-body, are strongly correlated with the hard X-ray flux (15-50 keV). We update the analytical description of these dependence, including a change in the photon index behaviour from a flat to a logarithmic function. The flux, where the change occurs, is consistent with the onset of the black-body emission. Thus, a change of the accretion state always occurs in GRO J1008-57 at a particular flux level.
\end{abstract}

\section{Motivation}

One of the remaining puzzles in our understanding of accreting neutron star binaries is the formation of their X-ray spectra and lightcurves. One has to deal with extreme physical conditions, such as high magnetic fields on the order of $10^{12} \mathrm{G}$, relativistic velocities, gravitational redshift and non-ideal plasmas. In the past decade, improvements of our understanding of accretion columns have started to reveal relations between observables and properties of the neutron star. It is of particular importance to support our understanding with comparisons between observations and theoretical milestones. Some examples are

\footnotetext{
ae-mail: matthias.kuehnel@sternwarte.uni-erlangen.de
}

This is an Open Access article distributed under the terms of the Creative Commons Attribution License 2.0, which permits unrestricted use, distribution, and reproduction in any medium, provided the original work is properly cited. 
- the connection between the spin-change of the neutron star caused by the angular momentum transfer [1],

- the influence of thermal and bulk Comptonization in the accretion column on the X-ray spectrum[2],

- the spectral formation of cyclotron resonant scattering features (CRSF) in a static accretion column $[3,4]$,

- the emission height of CRSF depending on the mass accretion rate [5].

All these theories predict that the observable quantities depend on the mass accretion rate, i.e., the Xray luminosity, of the neutron star. Thus, the best way to probe these theories is by studying sources varying over a wide luminosity range, which is the case during X-ray outbursts of transient neutron star X-ray binaries with a Be-type companion (BeXRB).

\subsection{Observational background}

Due to the complex physical processes, the observed X-ray spectra of accreting neutron stars cannot be described self-consistently by currently existing models. Therefore, the models used to successfully fit the data are, in most cases, of empirical nature (for a summary of commonly used models see [6] and [7]). To analyze the correlation of the spectral components with the luminosity, many observations of one particular source covering a wide flux range have to be analyzed. The strength and time properties (e.g., date and duration) of an outburst of a BeXRB mainly depend on the activity of the Be-type companion, i.e., on the size of its circumstellar disk $[8,9]$. Because its formation and behaviour are not understood in detail, the outburst dates of most BeXRBs are not predictable. Consequently, observations covering a wide luminosity range and, especially, the rising part of an outburst to check on hysteresis effects in the parameter evolution, are difficult to schedule.

The time-scale between outbursts of a source is either of the order of 10 to 100 days (around the periastron passage of the neutron star, leading to so-called type I outbursts), or can be many years (apparently not connected to any specific orbital phase, known as "giant" type II outbursts). As an observational consequence different outbursts of the same BeXRB are often observed by different Xray missions, each with individual detector responses and data reduction methods. Due to these time constraints detailed analyses of multi-outburst- and -mission-observations are rare. Fortunately, our timing analysis of the BeXRB GRO J1008-57 showed that its outburst times can be predicted with an accuray allowing to schedule observations during particular outburst phases [10].

\section{GRO J1008-57}

The neutron star in the transient high mass X-ray binary GRO J1008-57 orbits its Be-star companion of type B0e [11] with an eccentricity of 0.68 [12]. By analyzing the arrival times of the $93.7 \mathrm{~s}-$ pulsations as detected during outbursts in 2005 and 2007 by RXTE, Swift, and Suzaku, we were able to improve the time of the periastron passage and the orbital period to 249.48(4) $\mathrm{d}$ [10]. Using this ephemeris we calculated the orbital phases of all detected outbursts of GRO J1008-57 in RXTE-ASM and $S$ wift-BAT, which usually last for $14 \mathrm{~d}$. As a result almost all outbursts are consistent with the same orbital phase range close to periastron, which allows us to predict the time of maximum flux during outbursts of this source within $3 \mathrm{~d}[10]$.

Using this advantage, we asked for $R X T E$-observations during the rise of a predicted outburst of GRO J1008-57 in 2011 April. In addition to the RXTE pointings during the decays of the 2005 and 2007 outbursts, these data allow to study the spectral evolution of this BeXRB depending on outburst 
phase and luminosity state in great detail. The spectrum of GRO J1008-57 above $10 \mathrm{keV}$ can be well described by a power-law with high exponential cutoff [10, 12-14]. Here, we use the form

$$
\operatorname{CUTOFFPL}(E) \propto E^{-\Gamma} e^{-E / E_{\text {fold }}}
$$

where $\Gamma$ is the photon index, and $E_{\text {fold }}$ the folding energy. To describe the spectra below $10 \mathrm{keV}$ a black-body spectrum as well as an iron $\mathrm{K} \alpha$ fluorescence line at $6.4 \mathrm{keV}$ is added. Finally, all components are modified by interstellar absorption using the TBnew-model, a revised version of the absorption model of TBabs [15].

\subsection{X-ray Observations}

Recently, Reig \& Nespoli (2013) [14] and ourselves [10] studied the evolution of spectral parameters of GRO J1008-57 depending on its luminosity. In contrast to the first work, which used the 2007 RXTE-data only, we use one simultaneous Suzaku-pointing and one quasi-simultaneous $S$ wiftpointing during that outburst in addition as well as RXTE-monitoring during outbursts in 2005 and 2011. Because the RXTE-PCA had a field of view of $\sim 1^{\circ}$ and no imaging capabilities [16], the spectrum of a source near the Galactic plane, as is the case for GRO J1008-57, is contaminated by Galactic ridge emission (GRE; see, e.g., [17] and references therein). Using data from an RXTEcampaing in 1996/97, where GRO J1008-57 was in a quiescence state, we are able to remove the influence of the GRE from the source spectra using a model by $[18,19]$. In this way we are able to compare the spectral parameters between different outbursts and during the rise and decay. The log of all observations can be found in the online material ${ }^{1}$ of [10].

\subsection{Spectral Evolution}

The results of Reig \& Nespoli (2013) [14] show that the photon index $\Gamma$ as well as the folding energy $E_{\text {fold }}$ are correlated with source flux. However, they used data from RXTE-PCA only, covering the 3 to $60 \mathrm{keV}$ energy range. Our spectral fits (see [10]) using the same dataset in combination with Swift-XRT and Suzaku-XIS show that a black body component with a temperature of around $k T=$ $1.8 \mathrm{keV}$ is needed to successfully describe the spectrum below $3 \mathrm{keV}$. By investigating contour maps of different flux levels, we found that this temperature stays constant over the 2007 outburst within uncertainties. Further investigations show that the folding energy $E_{\text {fold }}$ stays constant over time as well. This is in contrast to earlier findings [14] but can be explained by the often observed statistical degeneracy between the photon index $\Gamma$ and the folding energy when there is not a broad (up to $\sim 100 \mathrm{keV}$ ) energy coverage.

To constrain the time-independent parameters $k T$ and $E_{\text {fold }}$ well, we performed a simultaneous fit of all available data of the 2007 outburst of GRO J1008-57 applying the method we described in [20]. Please note that compared to the usual approach, where each spectrum is fitted individually, we analyzed all spectra of the source simultaneously using the Interactive Spectral Interpretation System [ISIS, 21] and performed one combined fit. Thereby, a set of so-called group parameters, which are still allowed to vary between all spectra, is assigned to each spectrum. On the other hand, the time-independent global parameters are determined by all data during the $\chi^{2}$-minimization. After a successful fit of all spectra during the GRO J1008-57's 2007 outburst, we added the 2005 and 2011 spectra to the simultaneous fit to check if the global parameters have the same values during different outbursts and between rise and decay of an outburst.

\footnotetext{
${ }^{1}$ http: //www.aanda.org/articles/aa/full_html/2013/ 07/aa21203-13/T9.html
} 


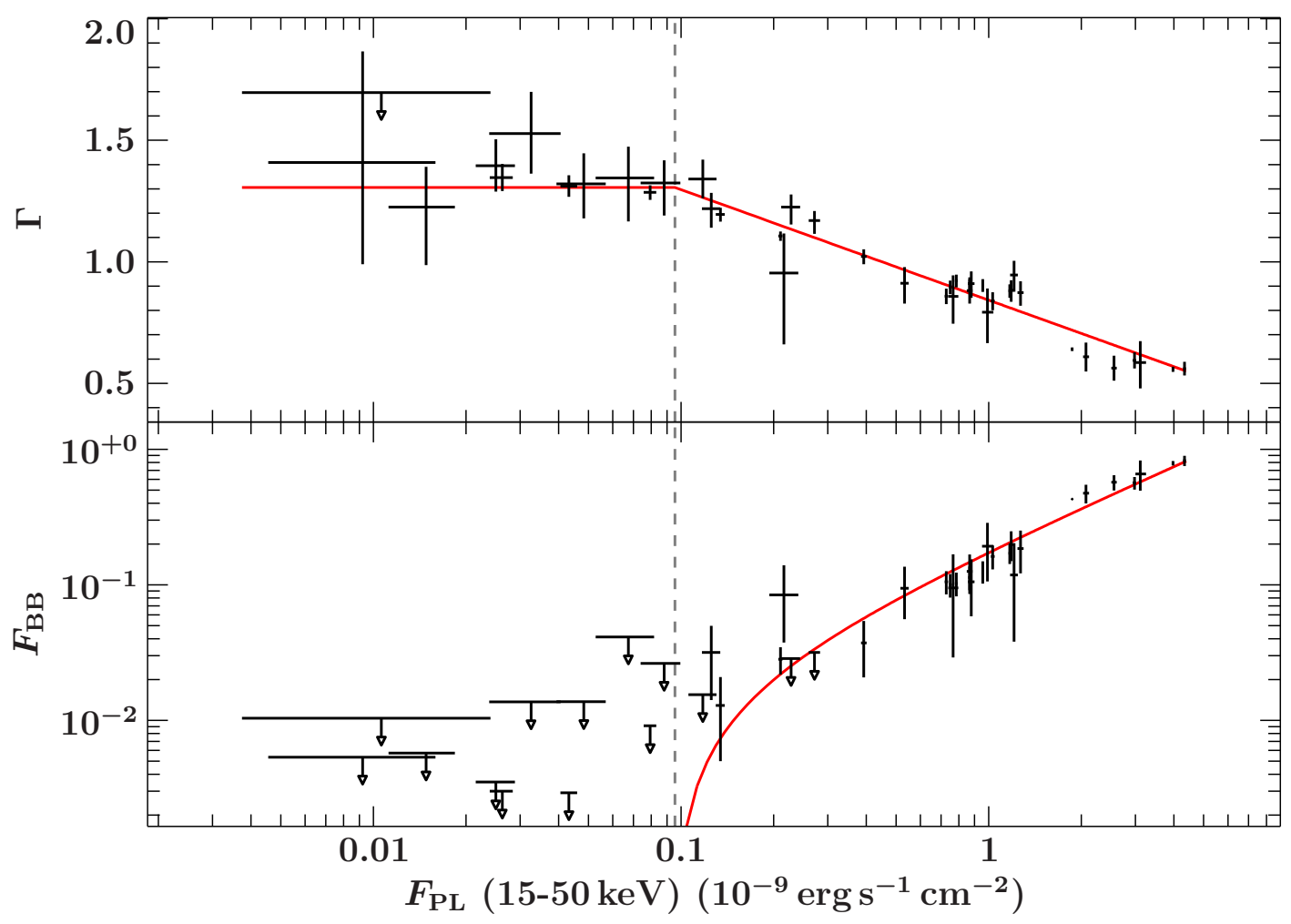

Figure 1. The photon index $\Gamma$ (top) and the black-body flux $F_{\mathrm{BB}}$ (bottom) as function of the power-law flux $F_{\mathrm{PL}}$ $(15-50 \mathrm{keV})$ as measured during outbursts of GRO J1008-57 in 2005, 2007, and 2011. The best-fit of empirical functions to the black data-points as described in the text is shown in red, while the dotted vertical line is the fitted flux level, where a change in the parameter correlations occurs.

As a remarkable result, the spectrum of GRO J1008-57, at any time, can be described by only three free parameters: the power-law flux $F_{\mathrm{PL}}$, the black-body flux $F_{\mathrm{BB}}$, and the photon index $\Gamma$ [22]. Their correlations are shown in Fig. 1 for each of the 68 analyzed observations. The photon index $\Gamma$ and black-body flux $F_{\mathrm{BB}}$ clearly correlate with the power-law flux $F_{\mathrm{PL}}(15-50 \mathrm{keV})$. Just as the global parameters, these correlations do not depend on a specific outburst or on the rise or decay. In [10], we fitted a logarithmic and a linear function to the $\Gamma$ - and $F_{\mathrm{BB}}$-correlation, respectively. Knowing the global parameters and the latter correlations, leads to the following result:

the spectrum of GRO J1008-57 can be described knowing only one parameter, which is the power-law flux.

To the authors' knowledge this is the first neutron star of which the spectrum can be predicted by using only the flux measured by an all-sky-monitor, e.g., by the Swift-BAT.

The simple fit to the correlations (Fig. 1) we performed in our previous analysis [10] lead to a good description of the data $\left(\chi_{\text {red }}^{2}=1.28\right.$ with 76 d.o.f. $)$. The linear equation describing the blackbody flux shows that the black-body significantly contributes once the power-law flux reaches $10(4) \times$ $10^{-11} \mathrm{erg} \mathrm{s}^{-1} \mathrm{~cm}^{-2}$. If we interpret this flux level as caused by a change in the accretion process 
onto the neutron star, the question arises whether there is evidence for a change in the photon index behaviour at the same flux level as well. Indeed, modifying its correlation with the source's flux (upper panel of Fig. 1), such that below a certain flux value the photon index is independent of the power-law flux, leads to a better description of the data than we obtained before. Furthermore, the flux level where that change appears is consistent with the onset of the black-body component. Thus, we performed a combined fit of both correlations with only one flux level, $F_{0}$, among the fit parameters, which results in a perfect description of the data $\left(\chi_{\text {red }}^{2}=1.02\right.$ with 76 d.o.f., see red line of Fig. 1). The improved parameter correlations compared to our previous analysis [10] are

$$
\Gamma= \begin{cases}\Gamma_{0} & , F_{\mathrm{PL}}<F_{0} \\ \Gamma_{0}-b \ln \left(\frac{F_{\mathrm{PL}}-F_{0}}{10^{-9} \mathrm{erg} \mathrm{s}^{-1} \mathrm{~cm}^{-2}}\right) & , F_{\mathrm{PL}} \geq F_{0}\end{cases}
$$

and

$$
F_{\mathrm{BB}}= \begin{cases}0 & , F_{\mathrm{PL}}<F_{0} \\ d\left(F_{\mathrm{PL}}-F_{0}\right) & , F_{\mathrm{PL}} \geq F_{0}\end{cases}
$$

with $F_{0}=0.96_{-0.22}^{+0.20} \times 10^{-10} \mathrm{erg} \mathrm{s}^{-1} \mathrm{~cm}, \Gamma_{0}=1.31 \pm 0.04, b=0.197_{-0.009}^{+0.008}$, and $d=0.190_{-0.010}^{+0.010}$.

\section{Conclusions}

The unexpected result of our analysis of three outbursts of GRO J1008-57 using different X-ray satellites, is that its spectrum can be predicted just knowing its $15-50 \mathrm{keV}$ flux. Above a certain flux level, which corresponds to an unabsorbed luminosity of $1.54_{-0.26}^{+0.22} \times 10^{36} \mathrm{erg} \mathrm{s}^{-1} \mathrm{~cm}$ between 0.01 and $100 \mathrm{keV}$ as predicted by our spectral model, the power-law as well as the black-body dependency on the source flux changes. That is in agreement with observational findings by [14], who discovered a change in parameter correlations in several BeXRB, and it confirms that some observable quantities of neutron stars, which is the spectral shape in case of GRO J1008-57, depend on the X-ray luminosity (compare Section 1). In particular, our findings can be interpreted in the context of accretion state changes as proposed by Becker et al. (2012) [5]. These authors investigated the X-ray emission height in accretion columns as a function of luminosity. They calculated luminosity levels, where in each case different forces are balanced. In particular, at a certain level the infalling material can be stopped by Coulomb interactions rather than by striking the surface of the neutron star. With increasing luminosity the height where the Coulomb interactions will decelerate the material to rest will increase as well. That might explain why the power-law index $\Gamma$ changes above the flux level we reported here. On the other hand, once the source is below that value the matter hits the surface directly and the spectral shape will not change with decreasing luminosity. However, this explanation must be treated with causion, because no physical model based on these theoretical hypotheses are implemented yet which could be fitted to observational data. Furthermore, other neutron star transients should show a similar behaviour in case the above picture turns out valid. Thus, further observations and thorough analysis are needed.

\section{Acknowledgements}

We thank the Bundesministerium für Wirtschaft und Technologie for support via Deutsches Zentrum für Luft- und Raumfahrt grant 50OR1113 and the Deutscher Akademischer Austauschdienst. The figure shown in this work was created with the SLXfig package developed by John E. Davis. 


\section{References}

[1] P. Ghosh, F.K. Lamb, ApJ 234, 296 (1979)

[2] P.A. Becker, M.T. Wolff, ApJ 654, 435 (2007)

[3] G. Schönherr, J. Wilms, P. Kretschmar, I. Kreykenbohm, A. Santangelo, R.E. Rothschild, W. Coburn, R. Staubert, A\&A 472, 353 (2007)

[4] F. Schwarm, G. Schönherr, J. Wilms, P. Kretschmar, Monte-Carlo Modeling of Cyclotron Resonant Scattering Features, in An INTEGRAL view of the high-energy sky (the first 10 years) (2012), PoS

[5] P.A. Becker, D. Klochkov, G. Schönherr, O. Nishimura, C. Ferrigno, I. Caballero, P. Kretschmar, M.T. Wolff, J. Wilms, R. Staubert, A\&A 544, A123 (2012)

[6] S. Müller, C. Ferrigno, M. Kühnel, G. Schönherr, P.A. Becker, M.T. Wolff, D. Hertel, F.W. Schwarm, V. Grinberg, M. Obst et al., 551, A6 (2013)

[7] M.E. DeCesar, P.T. Boyd, K. Pottschmidt, J. Wilms, S. Suchy, M.C. Miller, The Astrophysical Journal 762, 61 (2013)

[8] A.T. Okazaki, I. Negueruela, A\&A 377, 161 (2001)

[9] A.T. Okazaki, K. Hayasaki, Y. Moritani, PASJ (2012), in press (arXiv:1211.5225)

[10] M. Kühnel, S. Müller, I. Kreykenbohm, F. Fürst, K. Pottschmidt, R.E. Rothschild, I. Caballero, V. Grinberg, G. Schönherr, C. Shrader et al., A\&A 555, A95 (2013)

[11] M.J. Coe, P. Roche, C. Everall, J. Fabregat, D.A.H. Buckley, R.C. Smith, A.P. Reynolds, I.D. Jupp, H.T. MacGillivray, MNRAS 270, L57 (1994)

[12] M.J. Coe, A.J. Bird, A.B. Hill, V.A. McBride, M. Schurch, J. Galache, C.A. Wilson, M. Finger, D.A. Buckley, E. Romero-Colmenero, MNRAS 378, 1427 (2007)

[13] S. Naik, B. Paul, C. Kachhara, S.V. Vadawale, MNRAS 413, 241 (2011)

[14] P. Reig, E. Nespoli, A\&A 551, A1 (2013)

[15] J. Wilms, A. Allen, R. McCray, ApJ 542, 914 (2000)

[16] K. Jahoda, C.B. Markwardt, Y. Radeva, A.H. Rots, M.J. Stark, J.H. Swank, T.E. Strohmayer, W. Zhang, ApJS 163, 401 (2006)

[17] M. Revnivtsev, S. Sazonov, E. Churazov, W. Forman, A. Vikhlinin, R. Sunyaev, Nat 458, 1142 (2009)

[18] K. Ebisawa, S. Yamauchi, Y. Tanaka, K. Koyama, Suzaku Team, Progress of Theoretical Physics Supplement 169, 121 (2007)

[19] S. Yamauchi, K. Ebisawa, Y. Tanaka, K. Koyama, H. Matsumoto, N.Y. Yamasaki, H. Takahashi, Y. Ezoe, PASJ 61, 225 (2009)

[20] M. Kühnel, S. Müller, I. Kreykenbohm, F.W. Schwarm, S. Grossberger, T. Dauser, Ac. Polytechnica (2013), submitted

[21] J.C. Houck, L.A. Denicola, ISIS: An Interactive Spectral Interpretation System for High Resolution X-Ray Spectroscopy, in Astronomical Data Analysis Software and Systems IX, edited by N. Manset, C. Veillet, D. Crabtree (2000), Vol. 216 of Astron. Soc. of the Pacific Conf. Series, p. 591

[22] M. Kuehnel, S. Mueller, F. Fuerst, F. Schwarm, I. Kreykenbohm, J. Wilms, K. Pottschmidt, S. Suchy, R.E. Rothschild, I. Caballero et al., GRO J1008 57: a laboratory for accretion physics, in An INTEGRAL view of the high-energy sky (the first 10 years) (2012), PoS 